\title{
Weakness Cryptanalysis of Liao's Scheme and Improved Remote User Authentication Scheme for Mobile Device
}

\author{
Hie Do Kim ${ }^{1}$ and Kwang Cheul Shin ${ }^{2 *}$ \\ ${ }^{1}$ Department of Military Science(Naval Information Communication), Gang Neung \\ Yeong Dong College, \#357, Gong Je Ro, Gang Neung -Si, Kang Won-Do, 210-792, \\ South Korea \\ ${ }^{2}$ Division of Industrial Management Engineering, Sungkyul University, \#147-2, \\ Anyang 8 dong, Manan-gu, Anyang-si, Gyeonggi-do 430-742, Korea \\ ${ }^{1}$ skcskc12@sungkyul.ac.kr, ${ }^{2}$ hdkim@gyc.ac.kr
}

\begin{abstract}
Liao et al. 's has recently announced the suitable authentication scheme for mobile device environment, which can authenticate remote users by using $Q R$ code. However, Liao et al.'s scheme cannot satisfy several important security requirements. The biggest drawback of Liao et al.'s scheme is that it is not able to satisfy the mutual authentication between remote users and SP since it is vulnerable to eavesdropping, man-in-the-middle, theft and loss of mobile devices and forgery attack.

This paper aims to analyze the problems of Liao, et al., scheme and propose a safe authentication scheme using password based QR code that has fixed the aforementioned vulnerabilities.
\end{abstract}

Keywords: mobile device, authentication scheme, QR Code, impersonation attack

\section{Introduction}

The user authentication is essential for e-commerce and m-commerce in terms of accessing important resources. The user authentication protocol registers information that will allow users to confirm their identity from the servers providing services in advance. It also let users use services provided by the servers anytime and anywhere when they want to use services.

Recently, mobile devices are providing portability along with various functions such as PC, camera, recorder, etc., Due to the fact that it has become easy for anyone to produce and distribute mobile contents in an open environment, the likelihood of security threat is on the rise. QR (Quick Response) code based OTP (One Time Password) authentication protocol [1], eliminates the usage of the password verification table and also is a cost effective solution since most internet users already have mobile phones.

There are many advantages to use the QR code [2] in mobile phones such as Omnidirection readability and error correction capability [3]. In 2010, Liao, et al., proposed a user authentication scheme based on QR Code in which users may send a login request message without using their password [4].

Liao, et al., scheme does not utilize the password table and realize the cost efficiency by using the decryption and encryption algorithm and the hash function [5-7].

However, the reasons why Liao, et al., scheme cannot provide a perfect mutual authentication between users and SP (Service Provider) are as follows.

\footnotetext{
* Corresponding Author
} 
First, third parties are able to disguise themselves by tapping messages at the login phase for server access. Second, third parties are able to extract and use the information saved in mobile devices as though they are legitimate users after they stole and owned mobile devices.

Third, SP does not have any function to authenticate whether a message received from a user at the login phase is a legitimate message sent by users. Fourth, users do not have a function to verify whether third parties are performing the role of a server in disguise of SP. Fifth, for such results as mentioned, a safe mutual authentication between users and SP is lacking.

In this paper, we propose a safe mutual authentication scheme in mobile device environment, which complements the aforementioned drawbacks.

The proposed method is based on ownership (mobile device) and knowledge (password) factors. It combines the above factors challenge response methods without requiring any time synchronization or OTP between user's mobile phone and service provider's server for authentication purpose [8].

The rest of the paper is organized as follows section 2, 3 presents literature review, section 4 describes the motivation for the work and the contributions of this paper. Section 5 discusses the proposed scheme and its details. Section 6 presents the analysis of the scheme and finally Section 7 presents the conclusions.

\section{Review of Liao, et al.,'s Authentication Scheme}

This section reviews a user authentication scheme proposed by Liao, et al., The scheme participants include a remote user and a service provider. For simplicity, we denote the remote user $\mathrm{A}$ by $\mathrm{U}_{\mathrm{A}}$, and the service provider by SP.

The scheme assumes that each authorized user can request service from SP with granted access rights. In addition, each user hold a mobile phone with embedded camera, therefore he can take a picture of the QR code image and then decode it. Liao, et al., scheme consists of two phases: registration phase and authentication phase.

The registration phase is performed only once per user when a new user registers itself with the service provider. The authentication phase is carried out whenever a user wants to gain access to the service provider. Before the registration phase is performed for the first time, the service provider SP decides on the following system parameters: a one-way hash function $\mathrm{h}$ and a cryptographic key $\mathrm{s}$. The key $\mathrm{s}$ is kept securely the service provider. The notation is employed throughout this paper.

\section{Table 1. Notation and Description}

$U_{A}$ User A

$I D_{A}$ Identifier of User A

pwa Password of User A

$h(\cdot)$ An one-way hash function

$E_{Q R}(\cdot)$ A function that encodes data into QR code image

$D_{Q R}(\cdot)$ A function that decodes the QR code image captured in an embedded camera device $s$ SP's long term secret key

T1, T2 Time stamps

\| Concatenation operation

$\oplus$ XOR operation 


\section{A. Registration Phase}

Assume that a $U_{A}$ with an embedded camera mobile device wants to join the system. Then, $\mathrm{SP}$ and $\mathrm{U}_{\mathrm{A}}$ carry out the following registration procedures.

step $1: \mathrm{U}_{\mathrm{A}}$ sends his identity $\mathrm{ID}_{\mathrm{A}}$ to $\mathrm{SP}$.

step $2:$ SP computes

$\mathrm{X}_{\mathrm{A}}=\mathrm{h}\left(\mathrm{ID}_{\mathrm{A}} \| \mathrm{s}\right)$

and sends $\mathrm{X}_{\mathrm{A}}$ to $\mathrm{U}_{\mathrm{A}}$ 's mobile device via a secure channel.

step $3: U_{A}$ 's mobile device stores $X_{A}$ as the long term secret key.

\section{B. Verification Phase}

The verification phase is shown as follows.

step $1: \mathrm{U}_{\mathrm{A}}$ sends $\mathrm{ID}_{\mathrm{A}}$ and $\mathrm{T} 1$ to $\mathrm{SP}$, where $\mathrm{T} 1$ is the time stamp attached by the User A.

step 2 : SP examines whether the time stamp T1 is correct.

If it is invalid, then rejects it. Otherwise, he chooses a random number $r$, computes

$\mathrm{X}_{\mathrm{A}}=\mathrm{h}\left(\mathrm{ID}_{\mathrm{A}} \| \mathrm{s}\right)$, and $\alpha=\mathrm{r} \oplus \mathrm{X}_{\mathrm{A}}$

and then sends $E_{Q R}(\alpha), h(r\|T 1\| T 2)$, and $T 2$ to $U_{A}$, where $T 2$ is the time stamp attached by the SP.

step 3 : User A examines whether the time stamp T2 is correct. If it is invalid, then rejects it. Otherwise, he derives $r$ by computing

$\mathrm{r}=\mathrm{D}_{\mathrm{QR}}\left(\mathrm{E}_{\mathrm{QR}}(\alpha)\right) \oplus \mathrm{X}_{\mathrm{A}}$

with his embedded camera devices. After that, $\mathrm{U}_{\mathrm{A}}$ examines whether $\mathrm{h}(\mathrm{r}\|\mathrm{T} 1\| \mathrm{T} 2)$ is correct. If holds, then $\mathrm{U}_{\mathrm{A}}$ sends $\mathrm{h}(\mathrm{r}\|\mathrm{T} 2\| \mathrm{T} 3)$ and $\mathrm{T} 3$ to SP.

step 4 : SP examines whether the time stamp T3 is correct. If it is invalid, then rejects it. Otherwise, he checks whether $\mathrm{h}(\mathrm{r}\|\mathrm{T} 2\| \mathrm{T} 3)$ is correct. If holds, then SP is convinced that $\mathrm{U}_{\mathrm{A}}$ is validated. Otherwise, the request is rejected.

\section{Security Analysis of Liao et al.'s Scheme}

In this section, we will discuss the security drawbacks in Liao, et al.,'s QR code based remote user authentication Scheme. Since the message in login phase and verification phase are transmitted via an insecure channel, we assume that the third parties (attacker) can control the insecure channel.

\subsection{Man-in-the-middle Attack}

According to Sun, et al.,'s article(2009), the man-in-the-middle attack is defined as a form of active wiretapping attacks in which the attacker intercepts and selectively modifies communicated data in order to masquerade as one or more of the entities involved in a communication association [7].

Attackers are able to tap and read messages exchanged between $U_{A}$ and $S P . U_{A}$ is vulnerable to the main-in-the-middle attack since SP generates an authentication information $\alpha\left(r \oplus X_{A}\right)$ as to $U_{A}$ without any verification procedure when $U_{A}$ sends $\left\langle\mathrm{ID}_{\mathrm{A}} \| \mathrm{T} 1>\right.$ to $\mathrm{SP}$.

\subsection{Stolen Attack}

Assuming that $\mathrm{U}_{\mathrm{A}}$ lost a mobile device or a third parties stole a mobile device, third parties would be able to disguise themselves as a legitimate $\mathrm{U}_{\mathrm{A}}$ by extracting the confidential information $\left(\mathrm{X}_{\mathrm{A}}\right)$ saved in a mobile device. 
Third parties find out the random number $r$ generated by SP by decoding $\alpha$ through the decoder of a mobile device. Third parties own the confidential information $\mathrm{X}_{\mathrm{A}}$ and $\mathrm{r}$ of a legitimate $\mathrm{U}_{\mathrm{A}}$. Then, they generate $\mathrm{h}(\mathrm{r} 1\|\mathrm{~T} 2\| \mathrm{T} 3)$ and $\mathrm{T} 3$ in accordance with the following scenario and pass the authentication process by sending them to SP.

\subsection{User Spoofing Attack}

Assuming that third parties extract the secret value $\mathrm{X}_{\mathrm{A}}$ from the mobile device of a legitimate user, which they acquired through stolen attack, these third parties attempt to obtain an authentication after sending the log-in message $\left\langle\mathrm{ID}_{\mathrm{A}} \| \mathrm{T} 1\right\rangle$ to SP by disguising themselves as legitimate users. SP would verify the login message after receiving $\left\langle\mathrm{ID}_{\mathrm{A}} \| \mathrm{T} 1\right\rangle$.

Next, SP would generate and send $\operatorname{EQR}(\alpha), h(r\|T 1\| \mathrm{T} 2)$ and $\mathrm{T} 2$ to third parties. Third parties extract $r$ by computing $h(\operatorname{DQR}(\operatorname{EQR}(\alpha))$. Then, third parties obtain the time stamp $\mathrm{T} 3$ and send h(r || T2 || T3) to SP to get an approval as though they are legitimate users.

\subsection{Server Spoofing Attack}

Third parties that are in disguise of a server compute the following after receiving $<\mathrm{ID}_{\mathrm{A}} \| \mathrm{T} 1>$ from a legitimate $\mathrm{U}_{\mathrm{A}}$.

They select a random number $r^{\prime}$ and generate a random $X_{A}{ }^{\prime}$. Then, they calculate and send decode $\left(\mathrm{EQR}\left(\alpha^{6}\right)\right), \mathrm{h}\left(\mathrm{r}^{\prime}\|\mathrm{T} 1\| \mathrm{T} 2\right)$ on $\alpha^{\prime}$ from $\alpha^{6}=\mathrm{r}^{\prime} \oplus \mathrm{X}_{\mathrm{A}}{ }^{\prime}$ to $\mathrm{U}_{\mathrm{A}}$. $\mathrm{U}_{\mathrm{A}}$ calculates $\mathrm{r}^{\prime}\left(=\alpha^{\prime} \oplus \mathrm{X}_{\mathrm{A}}{ }^{\prime}\right)$ by using the confidential information $\mathrm{X}_{\mathrm{A}}$ as regarding it as a message sent from a legitimate SP. $\mathrm{X}_{\mathrm{A}}{ }^{\prime}$ is randomly generated by third parties; thus, the value of $\mathrm{r}^{\prime}$ must be trusted. $\mathrm{U}_{\mathrm{A}}$ authenticates third parties as SP since it trusts $r^{\prime}$ generated by third parties and generate and sends $h\left(r^{\prime}||\right.$ T2 || T3) and T3 to SP.

\section{Description of the Proposed Authentication Scheme}

In this section we proposed a password-based and use of the deployed widespread QR code in order to remote user authentication scheme which enhances on previous scheme.

The convenient integration of the web based application and the mobile devices usage makes our scheme more practical.

The proposed scheme involves two parties: a service provider (SP for short) and remote users. Each authorized user can request service from SP with the granted access rights. In addition, each user hold a mobile phone with embedded camera, therefore he can take a picture of the

QR code image and then decode it. Our scheme is divided into four phases: Registration, Login, Verification and Password change phases.

As mentioned earlier, our scheme improves over Liao et al.'s scheme in five ways:

(1) it does not require synchronized clocks between in the network by using password.

(2) it can withstand the password guessing attack even though the attacker has stolen some user's mobile device or gained access to it and extracted the secret values stored in it,

(3) it is secure against a service provider impersonation attack and a user impersonation attack, man in the middle attack and stolen attack.

(4) SP confirms whether $U_{A}$ 의 is a legitimate user by verifying the login messages of $U_{A}$, whereas $U_{A}$ 는 authenticates $S P$ by verifying whether $X_{A}$ can be generated. In this way, 2-way communication can be implemented. 
(5) The application module of a mobile device is operated only when password is entered. Therefore, it cannot be disguised even when it is stolen by third parties. Also, it allows for users to change the password whenever necessary.

There are four phases in our scheme including registration, login, authentication and password change phase. Detailed steps of these phases of the proposed scheme are described as follows.

\section{A. Registration Phase}

Before the $\mathrm{U}_{\mathrm{A}}$ logins to the SP, the user needs to perform the following steps.

Step 1: Firstly, $\mathrm{U}_{\mathrm{A}}$ send the $\mathrm{U}_{\mathrm{A}}$ 's ID, $\mathrm{ID}_{\mathrm{A}}$ to $\mathrm{SP}$ via a secure channel.

Step 2: Next, SP compute $X_{A}$ as follow:

$\mathrm{X}_{\mathrm{A}}=\mathrm{h}\left(\mathrm{ID}_{\mathrm{A}} \| \mathrm{s}\right)$, Where $\mathrm{s}$ is a secret key generated by the SP.

Step 3: Lastly, $S P$ sends $X_{A}$ to $U_{A}$ via a secure channel.

Step 4: $\mathrm{U}_{\mathrm{A}}$ chooses a password pwa and compute $\mathrm{hpwa}=\mathrm{h}\left(\mathrm{pwa} \| \mathrm{ID}_{\mathrm{A}}\right), \mathrm{A}=\mathrm{h}\left(\mathrm{X}_{\mathrm{A}}\right)$, and $\mathrm{B}=\mathrm{hpwa} \oplus \mathrm{A}$ on the mobile device.

Step 5: $\mathrm{U}_{\mathrm{A}}$ 's mobile device stores $\mathrm{B}$ as the long-term secret key.

\section{B. Login Phase}

Whenever the $\mathrm{U}_{\mathrm{A}}$ wants to login to the remote $\mathrm{SP}$, he/she must perform the following steps.

Step 1: When $\mathrm{U}_{\mathrm{A}}$ enters $\mathrm{ID}_{\mathrm{A}}$ and pwa, a mobile device confirms whether it is a legitimate user of a mobile device as the module application calculates the following. In other words, it verifies whether the password has been correctly entered.

$\mathrm{A}=\mathrm{B} \oplus \mathrm{h}\left(\mathrm{ID}_{\mathrm{A}} \| \mathrm{pwa}\right)=\mathrm{h}\left(\mathrm{h}\left(\mathrm{ID}_{\mathrm{A}} \| \mathrm{s}\right)\right.$

$\mathrm{B}^{\prime}=\mathrm{h}\left(\right.$ pwa $\left.\| \mathrm{ID}_{\mathrm{A}}\right) \oplus \mathrm{B}$

$\mathrm{A}=$ ? $\mathrm{B} \oplus \mathrm{h}\left(\right.$ pwa $\left.\| \mathrm{ID}_{\mathrm{A}}\right)$, accept or reject.

step 2: Calculate the following if it is a legitimate owner of a mobile device in step 1. random number ri, time stamp $\mathrm{T} 1$ select

$\mathrm{Ci}=\mathrm{h}\left(\mathrm{ID}_{\mathrm{A}}\|\mathrm{A}\| \mathrm{T} 1\right) \oplus \mathrm{ri}$, Where $\mathrm{T} 1$ is the time stamp attached by the $\mathrm{U}_{\mathrm{A}}$.

step 3 : At last, $\mathrm{U}_{\mathrm{A}}$ sends the message $\mathrm{ID}_{\mathrm{A}}, \mathrm{Ci}, \mathrm{T} 1$ to $\mathrm{SP}$.

\section{Authentication Phase}

After SP receiving the login message ( $\left.\mathrm{ID}_{\mathrm{A}}, \mathrm{Ci}, \mathrm{T} 1\right)$, Sp perform the following steps to mutual authentication.

Step 1: $\mathrm{ID}_{\mathrm{A}}, \mathrm{T} 1$ check. Reject or accept. SP examines whether the time stamp T1 is correct. If it is invalid, then reject it. Otherwise, he computes $X_{A}$ and ri.

$\mathrm{X}_{\mathrm{A}}=\mathrm{h}\left(\mathrm{ID}_{\mathrm{A}} \| \mathrm{s}\right), \mathrm{A}=\mathrm{h}\left(\mathrm{X}_{\mathrm{A}}\right)$.

$\mathrm{ri}=\mathrm{Ci} \oplus \mathrm{h}\left(\mathrm{ID}_{\mathrm{A}}\left\|\mathrm{h}\left(\mathrm{X}_{\mathrm{A}}\right)\right\| \mathrm{T} 1\right)$

After compute $X_{A}$ and ri, SP first verifies whether $C i=$ ? $h\left(I D_{A}\left\|h\left(X_{A}\right)\right\| T 1\right)$. If it holds, SP believes that $\mathrm{U}_{\mathrm{A}}$ is authenticated and then computes the following message to provide mutual authentication between $\mathrm{SP}$ and $\mathrm{U}_{\mathrm{A}}$.

Step 2: SP computes the follows:

$\alpha=\mathrm{h}\left(\mathrm{ri} \oplus \mathrm{h}\left(\mathrm{X}_{\mathrm{A}}\right) \| \mathrm{T} 2\right)$, Where T2 is the time stamp attached by the SP, compute $\mathrm{E}_{\mathrm{QR}}(\alpha)$.

Step 3: $\mathrm{SP}$ sends the message $\mathrm{E}_{\mathrm{QR}}(\alpha), \mathrm{T} 2$ to $\mathrm{U}_{\mathrm{A}}$.

Step 5: $\mathrm{U}_{\mathrm{A}}$ examines whether the time stamp $\mathrm{T} 2$ is correct. If it is invalid, then reject it. Otherwise, $\mathrm{U}_{\mathrm{A}}$ derives $\alpha$ by computing $\mathrm{D}_{\mathrm{QR}}\left(\mathrm{E}_{\mathrm{QR}}(\alpha)\right)=\mathrm{h}\left(\mathrm{ri} \oplus \mathrm{h}\left(\mathrm{X}_{\mathrm{A}}\right) \| \mathrm{T} 2\right)$ with his embedded camera device. 
After that, $\mathrm{U}_{\mathrm{A}}$ examines whether $\mathrm{h}\left(\mathrm{ri} \oplus \mathrm{h}\left(\mathrm{X}_{\mathrm{A}}\right) \| \mathrm{T} 2\right)$ is correct. If hold, then $\mathrm{U}_{\mathrm{A}}$ is convinced that $\mathrm{SP}$ is validated. Otherwise, the request is rejecting.

\section{Password Change Phase}

According to the above-mentioned requirements, $\mathrm{U}_{\mathrm{A}}$ can freely change the password, pwa, to a new password, pwa'. It can be finished without the help of the SP.

The steps of changing password are as follows.

step 1: Calculate $\mathrm{h}(\mathrm{A})$ after operating the password modification application by entering password.

$\mathrm{B}=\mathrm{B} \oplus \mathrm{h}\left(\right.$ pwa $\left.\| \mathrm{ID}_{\mathrm{A}}\right)=\mathrm{h}(\mathrm{A})$

Step 2: A mobile device calculates the following when a new password pwa' is entered.

$\mathrm{B}^{\prime}=\mathrm{h}(\mathrm{A}) \oplus \mathrm{h}\left(\mathrm{pwa}^{\prime} \| \mathrm{ID}_{\mathrm{A}}\right)$

Step 3: Replace B with B'

\section{Security Analysis of the Proposed Scheme}

We now analyze the security of the proposed scheme, considering password guessing attack, impersonation attacks, stolen mobile device attacks, man-in-the middle attack and providing proper mutual authentication.

In the Table 2-3, we compare the proposed scheme with previously published Liao et al.'s scheme.

\subsection{Resist Stolen Mobile Device Attack}

From Liao, et al., scheme, we have seen that a mobile device was used improperly when a legitimate user lost it or a third party acquired it. Third parties send $\left\langle\mathrm{ID}_{\mathrm{A}} \| \mathrm{T} 1\right\rangle$ by operating the remote authentication module in a mobile device of a legitimate user. SP that received a message does not have a function to verify whether it is a message sent from a legitimate user. SP calculates $\alpha=r \oplus X_{\mathrm{A}}$ by selecting a random number $\mathrm{r}$ and sends it along with $h(r\|T 1\| T 2)$ and $T 2$ to third parties after conducting encode $\left(\mathrm{E}_{\mathrm{QR}}(\alpha)\right)$ on $\alpha$.

Third parties own a mobile device of a legitimate user; therefore, they get an authentication by extracting $r$ by means of the saved $\mathrm{X}_{\mathrm{A}}$ and sending $\mathrm{h}(\mathrm{r}\|\mathrm{T} 2\| \mathrm{T} 3)$ and T3 to SP.

However, in the proposed paper, third parties are not able to find pwa of legitimate users and the confidential information $\mathrm{X}_{\mathrm{A}}$ of $\mathrm{SP}$ even after they picked up a mobile device. The information saved in a mobile device is $B\left(=h p w a \oplus h\left(X_{A}\right)\right)$; thus, it is not possible to calculate $h\left(X_{A}\right)$ without knowing the accurate password of users. Furthermore, $X_{A}$ calculates $B$ with the hash value; thus, it is safe in terms of loss and theft of a mobile device.

\subsection{Resist Man-in-the Middle Attack}

In our scheme, the remote server only has to maintain secret information, $s$, without storing the password tables. An attack may try to derive $\mathrm{X}_{\mathrm{A}}$ from the intercepted messages $\mathrm{ID}_{\mathrm{A}}, \mathrm{Ci}$, T1. But it is computationally infeasible because of the property of the one-way hashing function.

If the illegal user intercepts the message $\mathrm{ID}_{\mathrm{A}}, \mathrm{Ci}, \mathrm{T} 1$ from user $\mathrm{i}$ and try to masquerade as the remote server. It is impossible for the user to compute the message $\mathrm{Ci}$ unless he/she knows the secret information $\mathrm{X}_{\mathrm{A}}$ and $\mathrm{U}_{\mathrm{A}}$ 's password, pwa. 
An illegal user may try to fabricate fake request login messages to cheat the remote server into believing it is a legal remote login request (masquerade attack) in the login phase. It does not work unless he/she could modify Ci correctly.

Table 2. Comparison of Security Properties between Our Scheme and Liao, et al., Scheme

\begin{tabular}{|c|c|c|}
\hline & Liao, et al.,'s & Our proposed scheme \\
\hline Password guessing attack & N/A & resistance \\
\hline Impersonation attack & non-resistance & resistance \\
\hline Replay attack & resistance & resistance \\
\hline Man-in-the middle attack & non-resistance & resistance \\
\hline Stolen attack & non-resistance & resistance \\
\hline Password change & N/A & possible \\
\hline Proper mutual authentication & non-proper & proper \\
\hline
\end{tabular}

Table 3. Comparison of Computation and Communication between Our Scheme and Liao, et al., Scheme

\begin{tabular}{|c|c|c|}
\hline & Liao, et al.,'s & Our proposed scheme \\
\hline Registration & $1 \mathrm{Th}$ & $3 \mathrm{Th}$ \\
\hline Login & $1 \mathrm{Th}$ & $3 \mathrm{Th}$ \\
\hline Authentication & $4 \mathrm{Th}$ & $4 \mathrm{Th}$ \\
\hline Password change & $\mathrm{x}$ & $2 \mathrm{Th}$ \\
\hline Communication & 3-way & 2-way \\
\hline
\end{tabular}

\subsection{Resist Impersonation Attacks}

Impersonation attack is about an attacker who obtains a legitimate right by disguising him/herself as a legitimate user or SP after participating in the protocol.

Our scheme can resist two impersonation attacks, which are a service provider impersonation attack and a user impersonation attack.

An attacker cannot extract the confidential information $X_{A}$ within a mobile device. Moreover, he/she can no longer forge a valid response message $\langle\alpha\rangle$ or a valid login request message $<\mathrm{Ci}>$ although an attacker obtains the information (i.e., B) saved in a mobile device. A legitimate user utilizes hpwa $=\mathrm{h}\left(\mathrm{pwa} \oplus \mathrm{ID}_{\mathrm{A}}\right)$ in the registration phase; thus, insiders of SP or external third parties are not able to find it out. In order for a third party to disguise $\mathrm{him} /$ herself as an attacker, he/she should know the confidential information $\mathrm{X}_{\mathrm{A}}$ of SP.

However, it is saved along with user password in the form of $\mathrm{B}=\mathrm{hwpi} \oplus \mathrm{A}$ in a mobile device after SP conducted $A=h\left(X_{A}\right)$ on $X_{A}=h\left(I_{A} \| s\right)$ by the harsh value. In other words, it is safe since $\mathrm{B}$ cannot be extracted from $\mathrm{X}_{\mathrm{A}}$. In addition, third parties should be able to extract $X_{A}$ in order to disguise themselves as a server. If they are able to extract $X_{A}$, then they will be able to calculate a random number ri and generate legitimate $\alpha$. However, $X_{A}$ 는 is calculated by the hash value $h\left(X_{A}\right)$ and saved as the value of $B$ in a mobile device. Thus, it is safe for user/server spoofing attacks since it is not able to generate legitimate $\alpha$. 


\subsection{Proper Mutual Authentication}

The proposed scheme can provide proper mutual authentication. $U_{A}$ sends the message $\left(\mathrm{ID}_{\mathrm{A}}\right.$, $\mathrm{Ci}, \mathrm{T} 1)$ to SP. SP first check the validity of $\mathrm{U}_{\mathrm{A}}$ 's ID, $\mathrm{ID}_{\mathrm{A}}$, time stamp $\mathrm{T} 1$, then compute $\mathrm{X}_{\mathrm{A}}=\mathrm{h}\left(\mathrm{ID}_{\mathrm{A}} \| \mathrm{s}\right), \quad \mathrm{A}=\mathrm{h}\left(\mathrm{X}_{\mathrm{A}}\right) \quad$ and $\mathrm{ri}=\mathrm{Ci} \oplus \mathrm{h}\left(\mathrm{ID}_{\mathrm{A}}\left\|\mathrm{h}\left(\mathrm{X}_{\mathrm{A}}\right)\right\| \mathrm{T} 1\right)$, compare the compute $\mathrm{h}\left(\mathrm{ID}_{\mathrm{A}} \oplus \mathrm{h}\left(\mathrm{X}_{\mathrm{A}}\right) \oplus \mathrm{T} 1\right)$ value with the receiving $\mathrm{Ci}$ value. If they are equal, $\mathrm{SP}$ is a valid $\mathrm{U}_{\mathrm{A}}$. $\mathrm{U}_{\mathrm{A}}$ believes that $\mathrm{SP}$ is able to calculate $\mathrm{A}$ easily as long as it is legitimate. $\mathrm{U}_{\mathrm{A}}$ authenticates that $\mathrm{SP}$ is legitimate when the value of $\alpha$ matches the value calculated by $\mathrm{U}_{\mathrm{A}}$ after $\mathrm{A}$ decoded the value of $\mathrm{E}_{\mathrm{QR}}(\alpha)$ by the value that was hashed as a result of connecting the secret key s of SP to $\mathrm{ID}_{\mathrm{A}}$.

\subsection{Free Password Change}

If the legal user lost his/her mobile device, it is difficult for any adversary to derive or change the password because he/she cannot pass the password verification. The illegal request will be rejected.

\section{Performance Comparisons and Functionality Analysis}

In this section, we evaluate the performance and functionality of our proposed scheme and make comparisons with Liao, et al., scheme. To analyze the computational complexity of the schemes, we define the notation Th as the time complexity for hashing function. Because exclusion-OR operation requires very few computations, it is usually negligible considering its computational cost.

Table 3 shows the Performance Comparison of our scheme and Liao, et al., scheme. As you can see Table 3, In Liao, et al., scheme, the computation cost of registration phase, login phase, and authentication phase are 1Th, 1Th and 4Th, respectively. In our improved scheme, the computation cost of registration phase, login phase, and authentication phase are 3Th, 3Th and $4 \mathrm{Th}$, respectively. But our scheme can achieve the proper mutual authentication and can resist man-in-the middle attack, stolen attack, impersonation attack etc., in Table 2.

\section{Conclusion}

This paper reviewed and analyzed the authentication scheme of Liao et al. that was based on QR code. As a result of the analysis, it was found that Liao, et al., authentication scheme was vulnerable to several attacks of a third party. In conclusion, Liao, et al., authentication scheme does not provide a safe authentication between users and SP.

This paper proposed an enhanced authentication scheme using password based QR code for prevention of theft and spoofing attack of mobile devices and safe mutual authentication.

The differentiated point of the proposed paper from Liao, et al., authentication scheme is that users hash their password and the confidential information $\mathrm{X}_{\mathrm{A}}$ of SP in the registration phase and save them in a mobile device and consequently, users are able to select and renew a random password. Therefore, it can resist spoofing attacks, loss and theft associated attacks and man-in-the-middle attacks toward users and SP and also reduce the communication traffic for mutual authentication from three times to two times.

\section{References}

[1] K. C. Liao and W.-H. Lee, "A Novel User Authentication Scheme Based on QR-Code", Journal of Networks, vol. 5, no. 8, (2010) August 8, pp. 937-941.

[2] A. S. Narayanan, "QR Codes and Security Solutions", International Journal of Computer Science and Telecommunications, vol. 3, Issue 7, (2012) July. 
[3] N. Harini and Dr. T. R Padmanabhan, "A New Two Factor Authentication Scheme Using QR-Code", International of Engineering and Technology, vol. 5, no. 2, (2013) May, pp. 1087-1094.

[4] J.-S. Wang, F.-Y. Yang and I. Paik, "A Novel E-cash Payment Protocol Using Trapdoor Hash Function on Smart Mobile Devices", International Journal of Computer Science and Network Security, vol. 11, no. 6, (2011), pp. 12-19.

[5] Y. Lee, J. Kim, W. Jeon and D. Won. "Design of a Simple User Authentication Scheme Using QR-Code for Mobile Device", Information Technology Convergence, Secure and Trust Computing, and Data Management Lecture Notes in Electrical Engineering, vol. 180, (2012), pp. 241-247.

[6] T. Falas and H. Kashani, "Two-Dimensional Bar-code Decoding with Camera-Equipped Mobile Phones," Proceedings of the Fifth Annual IEEE International Conference on Pervasive Computing and Communications Workshops, (2007) March 19-23, pp. 597-600.

[7] X. Li, J.-W. Niu, J. Ma, W.-D. Wang and C.-L. Liu, "Cryptanalysis and improvement of a biometrics-based remote user authentication scheme using smart cards", Journal of Network and Computer Applications, vol. 34, (2011) pp. 73-79.

[8] "OTP Authenticators", Retrieved, (2009) November, from: http://www.safenetinc.com/Products /Data_Protection/Multi-Factor_Authentication/OTP_Authenticators.aspx.

[9] M. Kim, B. Lee, S. Kim and D. Won, "Weaknesses and Improvements of a One-time Password Authentication Scheme", International Journal of Future Generation Communication and Networking, vol. 2, no. 4, (2009) December.

[10] W. C. Kuo, Y. C. Lee, "Attack and improvement on the one-time password authentication protocol against theft attacks", Proc. of the Sixth International Conference on Machine Learning and Cybernetics, Hong Kong, (2007) August, pp. 19-22.

\section{Authors}

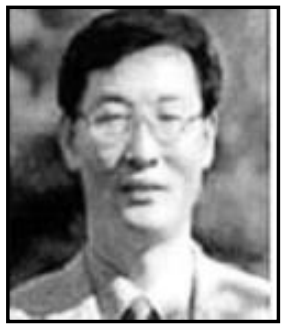

Hie Do Kim, Department of Military Science (Naval Information Communication), Gang Neung Yeong Dong College, \#357, Gong Je Ro, Gang Neung -Si, Kang Won-Do, 210-792, South Korea, hdkim@gyc.ac.kr Education \& Work experience: 2007, Ph.D. degree in Information and Communication Security Engineering, Sungkyunkwan University. Currently: Professor in Dept. of Military Science, Gang Neung Yeong Dong College. Tel: 82-033-610-0316.

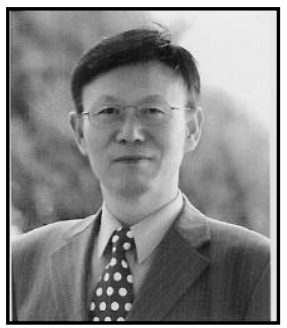

Kwang Cheul Shin, Division of Industrial Management Engineering, Sungkyul University, \#147-2, Anyang 8 dong, Manan-gu, Anyang-si, Gyeonggi-do 430-742, Korea, skcskc12@sungkyul.edu education \& Work experience: 2003, Ph.D. degree in Information and Communication Engineering, Sungkyunkwan University. Currently : Professor in Dept. of Industrial Management Engineering, Sungkyul University. Tel: 82031-467-8916. 
International Journal of Security and Its Applications Vol.8, No.5 (2014) 